\title{
Glycine targets NINJ1-mediated plasma membrane rupture to provide cytoprotection
}

Jazlyn P. Borges ${ }^{1, *}$, Allen Volchuk ${ }^{2, *}$, Bridget Kilburn ${ }^{1}$, Neil M. Goldenberg ${ }^{2,3,4}$, Benjamin E. Steinberg ${ }^{1,3,4}$

1. Program in Neuroscience and Mental Health, Hospital for Sick Children, Toronto, Ontario, Canada

2. Program in Cell Biology, Hospital for Sick Children, Toronto, Ontario, Canada

3. Department of Anesthesia and Pain Medicine, Hospital for Sick Children, Toronto, Ontario, Canada

4. Department of Anesthesiology and Pain Medicine, University of Toronto, Toronto, Ontario, Canada

* Equally contributing first authors

Corresponding author:

Benjamin E. Steinberg

Peter Gilgan Centre for Research and Learning

Hospital for Sick Children

Conflict of interest statement: The authors have declared that no conflict of interest exists. 
bioRxiv preprint doi: https://doi.org/10.1101/2021.12.12.471765; this version posted December 12, 2021. The copyright holder for this preprint (which was not certified by peer review) is the author/funder, who has granted bioRxiv a license to display the preprint in perpetuity. It is made available under aCC-BY-NC-ND 4.0 International license.

\section{Graphical abstract}

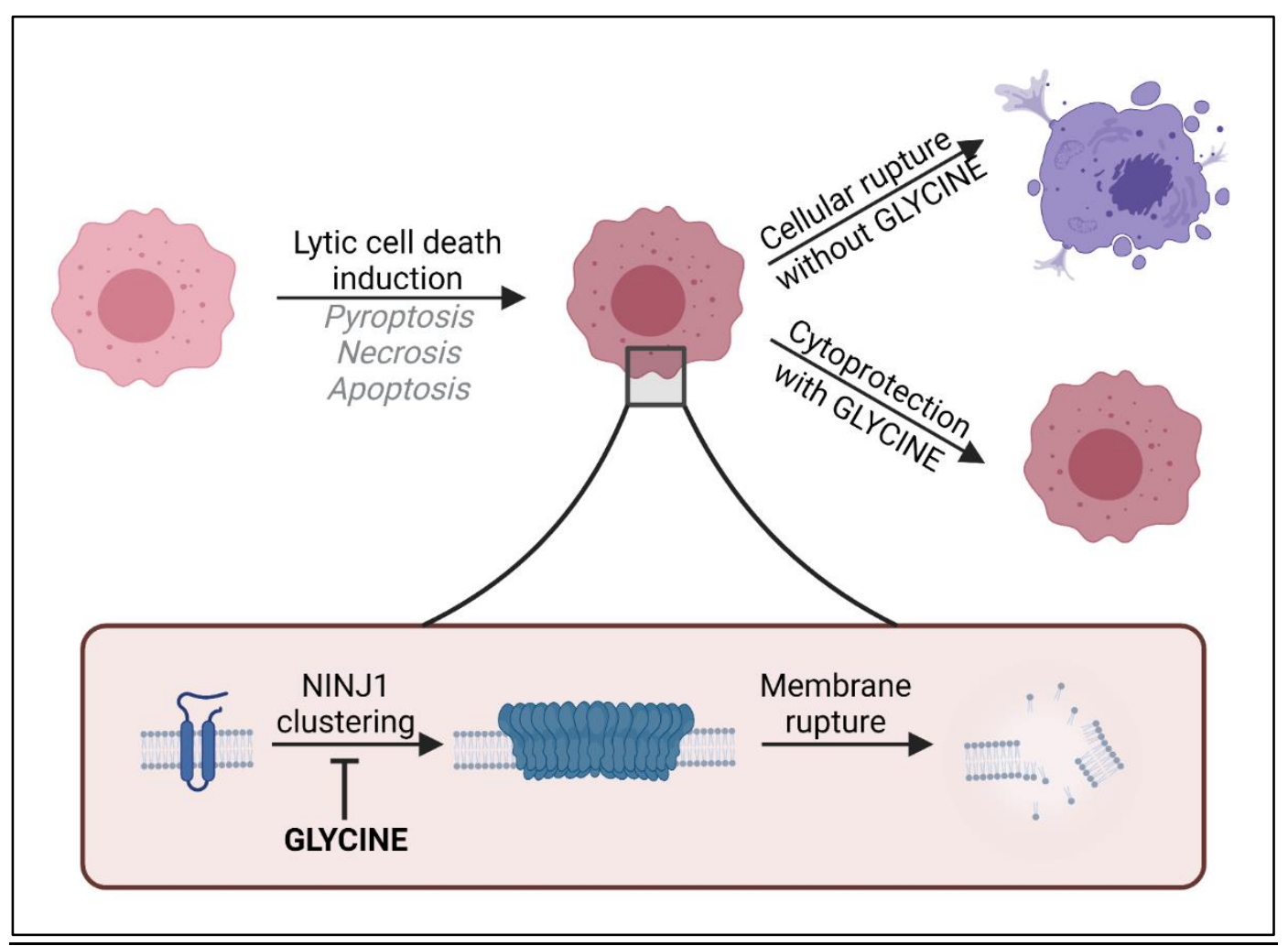




\section{$\underline{\text { Abstract }}$}

First recognized more than 30 years ago, glycine is known to protect cells against plasma membrane rupture from diverse types of tissue injury. This robust and widely observed effect has been speculated to target a late downstream process common to multiple modes of tissue injury. The molecular target and mechanism of glycine cytoprotection, however, remain entirely elusive. We hypothesized that glycine targets ninjurin-1 (NINJ1), a newly identified executioner of plasma membrane rupture in pyroptosis, necrosis, and apoptotic cell death. This common terminal effector is thought to cluster within the plasma membrane to cause cell rupture. Here, we first demonstrate that NINJ1 knockout functionally and morphologically phenocopies glycine cytoprotection in macrophages stimulated to undergo lytic cell death. Glycine treatment in NINJ1 knockout cells provides no additional protective effect. Next, we show that glycine treatment prevents NINJ1 clustering within the plasma membrane thereby preserving its integrity. By identifying NINJ1 as a glycine target, our data help resolve the longstanding mechanism of glycine cytoprotection. This new understanding will inform the development of cell and tissue preservation strategies for pathologic conditions associated with lytic cell death pathways. 


\section{Introduction}

The amino acid glycine has long been known to protect cells against plasma membrane rupture induced by a diverse set of injurious stimuli. This cytoprotective effect was first described in a report in 1987 where glycine was found to protect renal tubular cells against hypoxic injury (1). Since, similar observations have been replicated across immune, parenchymal, endothelial and neuronal cell types in multiple injury models [reviewed in (2)]. These include the lytic cell death pathways pyroptosis $(3,4)$ and necrosis (5).

While the robust cytoprotective effects of glycine are widespread, the molecular target and mechanism by which glycine protects cells and tissues remain elusive. Many cellular processes have been investigated but ultimately ruled out as underlying this phenomenon (2). These include glycine or ATP metabolic pathways (6), intracellular calcium or $\mathrm{pH}$ regulation (7), cytoskeleton stabilization (8), and chloride conductance (3). It has been speculated that glycine targets a membrane receptor but is unlikely to involve canonical glycine receptors $(2,3)$. Lastly, as a cytoprotective agent, glycine has oftentimes been described as an osmoprotectant, but its small size generally allows free passage through the large membrane conduits involved in lytic cell death pathways, such as the gasdermin D pores formed during pyroptosis (9), thereby negating a protective osmotic effect. A mechanistic knowledge of how glycine preserves cellular integrity, however, would inform the development of cell and tissue preservation strategies with clear clinical implications, such as for ischemia-reperfusion injuries where multiple lytic cell death pathways are activated to cause tissue damage and morbidity (10).

Given how widely observed the phenomenon is, it stands to reason that glycine targets a late downstream process common to multiple tissue injury models. Notably, the transmembrane protein 
ninjurin-1 (NINJ1) was recently identified as the common executioner of plasma membrane rupture in pyroptosis, necrosis, and post-apoptosis lysis (11). Post-apoptosis lysis refers to the cell membrane rupture that occurs when phagocytes are unable to scavenge apoptotic cells that have otherwise completed the apoptotic program (12). NINJ1 is a 16-kilodaltons protein with two transmembrane regions that resides in the plasma membrane and is reported to function as a cell adhesion molecule $(13,14)$. It was first identified for its role in nerve injury $(14)$ and has since been implicated in multiple inflammatory conditions, such as atherosclerosis, ischemic brain injury and tumorigenesis (15-17). Its involvement in lytic cell death pathways involves its clustering within the plasma membrane, which results in cellular disruption by an unknown mechanism.

Here, we hypothesized that glycine targets NINJ1 to mediate its cytoprotective effect. We first demonstrate that NINJ1 knockout functionally and morphologically phenocopies glycine cytoprotection in macrophages stimulated to undergo various forms of lytic cell death. Next, we show that glycine treatment prevents NINJ1 clustering within the plasma membrane thereby preserving its integrity. By identifying NINJ1 as a glycine target, our data help resolve the long-standing mechanism of glycine cytoprotection.

\section{$\underline{\text { Results and Discussion }}$}

NINJ1 knockout functionally and morphologically phenocopies glycine cytoprotection.

The published literature suggests a potential association between NINJ1 and glycine given that NINJ1 knockout and glycine treatment protect cells against a common set of lytic cell death pathways. Moreover, in pyroptosis, both allow for IL-1 $\beta$ secretion through the gasdermin D pore while preventing final membrane lysis $(4,11)$. We first sought to extend these associations by further characterizing the 
functional and morphological similarities between NINJ1 knockout and glycine cytoprotection in pyroptosis, necrosis and post-apoptosis lysis.

Using a CRISPR-Cas9 system, we generated a NINJ1 knockout cell line in immortalized bone marrowderived macrophages (iBMDM) (Figure 1A). Pyroptosis was induced in LPS-primed wildtype and NINJ1 knockout iBMDM with nigericin ( $20 \mu \mathrm{M}, 30 \mathrm{~min})$ in the absence or presence of $5 \mathrm{mM}$ glycine. We performed a colorimetric assay to test for lactate dehydrogenase (LDH) release, a common marker of cell rupture. Both NINJ1 knockout and glycine treatment in wildtype iBMDM protected against cytotoxicity (Figure 1B). Similarly, cell membrane integrity was preserved following apoptosis (Figure 1C) and necrosis (Figure 1D) induced with venetoclax ( $25 \mu \mathrm{M}, 16 \mathrm{~h})$ and pneumolysin $(0.5 \mathrm{ug} / \mathrm{ml}, 15 \mathrm{~min})$, respectively. Importantly, in NINJ1 knockout macrophages, treatment with glycine during lytic cell death did not confer any additional protection against cell lysis (Figure 1B-D).

When visualizing glycine-treated pyroptotic macrophages, we observed prominent plasma membrane ballooning (Figure 1E). This impressive morphology was comparable to that of LPS-primed NINJ1 knockout macrophages stimulated to undergo pyroptosis with nigericin, which we (Figure 1E) and others (11) have observed. Together, these functional and morphologic similarities buttress the association between glycine cytoprotection and NINJ1-mediated plasma membrane rupture.

Glycine targets NINJ1 clustering to prevent membrane rupture.

We therefore next posited that glycine targets NINJ1 as part of its cytoprotective effect. The working model of NINJ1-mediated membrane rupture involves its clustering within the plasma membrane (11). Point mutants (e.g. K45Q and A59P) that prevent membrane rupture also fail to cluster upon induction of pyroptosis (11) further reinforcing a connection between the clustering process and the capacity of 
NINJ1 to rupture membranes. To establish whether glycine directly interferes with NINJ1-mediated cell death, we next evaluated the effect of glycine treatment on NINJ1 clustering during cell lysis in pyroptosis, post-apoptosis, and necrosis.

Using a biochemical native-PAGE approach that maintains native protein interactions, endogenous NINJ1 of otherwise untreated cells migrates at approximately $40 \mathrm{kDa}$, potentially indicative of NINJ1 dimers or trimers in unstimulated cells (Figure $\mathbf{2 A}$ ) and consistent with its known ability to form homotypic interactions (18). In response to pyroptosis (nigericin in LPS-primed BMDM), necrosis (pneumolysin), and apoptosis (venetoclax) induction in primary bone marrow-derived macrophages (BMDM), the endogenous NINJ1 signal shifts to a high molecular weight aggregate, suggestive of a clustering process. Importantly, this shift is completely abrogated by glycine treatment (Figure $\mathbf{2 A}$ ). To corroborate these biochemical findings, we proceeded to evaluate native NINJ1 oligomerization by fluorescence spinning disk microscopy in primary BMDM induced to undergo pyroptosis. In unstimulated (not shown) and LPS-primed cells (Figure 2B), NINJ1 primarily localizes to the plasma membrane with some additional signal within the Golgi. Following induction of pyroptosis with nigericin in LPS-primed BMDM, NINJ1 redistributed into discrete puncta, which is consistent with clustering. Cotreatment with glycine prevented NINJ1 redistribution (Figure 2B).

Next, to confirm the plasma membrane localization of the observed puncta, we conducted total internal reflection (TIRF) microscopy of primary BMDM induced to undergo pyroptosis without or with glycine. TIRF microscopy allows for the preferential excitation of a thin $(\sim 150 \mathrm{~nm})$ layer that includes the ventral plasma membrane of adherent cells, thereby eliminating background fluorescence from structures outside this focal plane (e.g. endomembrane compartments). In LPS-primed BMDM, NINJ1 appears in discrete, small puncta that are diffusely distributed across the plasma membrane (Figure $\mathbf{2 C}$ ), consistent 
with the dimers or trimers predicted to exist in the basal state based on the native PAGE (Figure 2A).

Upon induction of pyroptosis, the density of these puncta decreases (Figure 2C-D), consistent with the clustering or aggregation of the basal dimers or trimers into high-order oligomers. Co-treatment of glycine abrogates the formation of these larger clusters (Figure 2C-D).

\section{Discussion.}

The protective effect of glycine treatment is a pervasive feature of many cell death pathways, but with an unknown mechanism of action. Here, we position the transmembrane protein NINJ1 as a component of the elusive glycine target.

In our studies, we focused on three fundamental cell death pathways in macrophages and show that glycine prevents NINJ1 clustering, a process required for plasma membrane rupture. Necroptosis represents another programmed cell death pathway, important to inflammatory responses, pathogen detection and tissue repair (19). Unlike pyroptosis, necrosis and post-apoptosis lysis, NINJ1 does not appear to be needed for necroptosis-induced plasma membrane rupture (11), potentially due to the membrane disrupting capacity of the necroptosis MLKL pore (20). This is particularly notable as glycine does not prevent TNF-induced necroptosis (21), further buttressing the association between NINJ1 and glycine.

Our data demonstrate that glycine treatment and genetic NINJ1 ablation yield similar functional and morphological outcomes in macrophages subjected to lytic cell death programs. Mechanistically, our data suggest that glycine cytoprotection results from interference with NINJ1 clustering within the plasma membrane. This finding is further supported by the observation that glycine treatment of NINJ1 knockout cells confers no additional protection against cellular rupture. Future work should be directed 
at determining whether glycine directly or indirectly engages NINJ1. Indeed, how NINJ1 is activated by lytic cell death pathways, its clustering trigger, and method of plasma membrane disruption also remain important and outstanding questions in the field. Due to the ubiquity of lytic cell death pathways in human health and disease and the potency of glycine cytoprotection, answers to these questions will greatly advance the development of therapeutics against pathologic conditions associated with aberrant lytic cell death pathways.

\section{Methods}

\section{Cells}

Primary bone marrow derived macrophages (BMDM) were harvested from the femurs and tibia of wildtype mixed-sex cohorts of $\mathrm{C} 57 \mathrm{BI} / 6$ mice. The ends of cleaned bones were cut and centrifuged to collect bone marrow into sterile PBS. The cell suspension was washed with PBS and plated in DMEM with $10 \mathrm{ng} \mathrm{mL}^{-1} \mathrm{M}$-CSF (Peprotech Inc, 315-02). Following 5 days of culture, the BMDM were detached from the dishes with TBS with 5 mM EDTA, resuspended in fresh DMEM and plated. Immortalized BMDM expressing Cas9 were obtained from Dr Jonathan Kagan (Boston Children's Hospital, Boston) and described previously (22).

NINJ1 knock-out cell line generation

iBMDM cells were transfected with custom CRISPR gRNA plasmid DNA (U6-gRNA:CMV-Cas-9-2A-tGFP)

(Sigma-Aldrich) using FuGENE HD transfection reagent (Promega). The NINJ1 target region sequence was GCCAACAAGAAGAGCGCTG. Twenty-four hours later the cells were FACS sorted for GFP into 96 well plates. Individual colonies were expanded and tested for NINJ1 expression by western blot.

Pyroptosis, apoptosis, and necrosis induction 
Pyroptosis was activated in macrophages primed with $(0.5 \mu \mathrm{g} / \mathrm{ml})$ LPS from E. coli serotype 055:B5, which was first reconstituted at a stock concentration of $1 \mathrm{mg} / \mathrm{ml}$. In primary BMDM, after $4.5 \mathrm{~h}$ of LPS priming, pyroptosis was induced with $20 \mu \mathrm{M}$ nigericin (Sigma N7143; stock $10 \mathrm{mM}$ in ethanol) for 30 min. Immortalized BMDM were primed with LPS for $3 \mathrm{~h}$, followed by pyroptosis induction with $10 \mu \mathrm{M}$ nigericin for $2 \mathrm{~h}$. Apoptosis was induced in non-primed macrophages using the $\mathrm{Bcl}-2$ inhibitor venetoclax (ABT-199, Tocris 6960) at $25 \mu \mathrm{M}$ for $16 \mathrm{~h}$. Necrosis was induced by treating non-primed macrophages with pneumolysin $(0.5 \mathrm{mcg} / \mathrm{ml})$ for $15 \mathrm{~min}$ in iBMDM or $45 \mathrm{~min}$ in primary BMDM. Pneumolysin was obtained from Dr John Brumell (Hospital for Sick Children, Toronto).

\section{LDH assay}

Cells were seeded at 200000 cells per well in 12-well plates, treated as indicated, and cytotoxicity assayed by LDH release assay the following day. At the end of the incubations, cell culture supernatants were collected, cleared of debris by centrifugation for $5 \mathrm{~min}$ at $500 \times \mathrm{g}$. The cells were washed once with PBS then lysed in lysis buffer provided in the LDH assay kit (Invitrogen C20300). Supernatants and lysates were assayed for LDH using an LDH colorimetric assay kit as per the manufacturer's instructions (Invitrogen C20300).

\section{Fluorescence microscopy}

Macrophages were cultured on $18 \mathrm{~mm}$ glass coverslips in 12-well plates at 150000 cells per well. Cell were treated as indicated in the figure legends, washed with PBS, fixed in $4 \%$ paraformaldehyde in PBS at room temperature for $15 \mathrm{~min}$, and permeabilized with 0.1\% Tween-20. Cells were then blocked in PBS supplemented with $10 \%$ donkey serum and $0.1 \%$ Tween- 20 for $1 \mathrm{~h}$ at room temperature prior to overnight incubation with primary antibody at $4{ }^{\circ} \mathrm{C}$. Rabbit monoclonal anti-mouse NINJ1 was used at 10 $\mathrm{mcg} / \mathrm{ml}$. Next, cells were washed three times with PBS supplemented with $10 \%$ donkey serum prior to 
the addition of secondary antibody at room temperature for $1 \mathrm{~h}$. Nuclei were labeled using DAPI containing mounting medium (ProLong Diamond Antifade Mountant, Invitrogen P36961). Cells were imaged by spinning disk confocal microscopy (Quorum) on a Zeiss Axiovert 200M microscope with a 63x objective and an additional 1.5x magnifying lens. Images were acquired by a CCD camera (Hamamatsu Photonics) using Volocity acquisition software. For total internal reflection microscopy, cells were costained with recombinant Cholera toxin subunit B conjugated to Fluor488 (Invitrogen C34775) at 1 $\mathrm{mcg} / \mathrm{mL}$ for less than 1 minute prior to fixing and staining for NINJ1 as described above. The Cholera toxin subunit B served as a membrane marker to optimize plasma membrane visualization. TIRF imaging was conducted on a Zeiss Axio Observer Z1 microscope with a 100x objective and Andor iXon3 885 detector. Rabbit monoclonal and polyclonal anti-mouse NINJ1 were obtained from Drs Nobuhiko Kayagaki and Vishva Dixit (Genentech Inc, San Francisco).

\section{Native and SDS-PAGE}

Macrophages were lysed with native-PAGE lysis buffer (150 mM NaCl, 1\% Digitonin, 50 mM Tris pH7.5, and $1 \times$ Complete Protease Inhibitor). Following centrifugation at $20800 \times \mathrm{g}$ for $30 \mathrm{~min}$, lysates were mixed with 4X NativePAGE sample buffer and Coomassie G-250 (ThermoFisher) and resolved using NativePAGE 3-12\% gels. For SDS-PAGE, cells were washed with 1x PBS and lysed in RIPA lysis buffer containing protease inhibitors (Protease inhibitor tablet, Pierce A32955). Proteins were resolved using NuPAGE 4-12\% Bis-Tris gels (Invitrogen), transferred to PVDF membranes and immunoblotted. GAPDH (Santa Cruz, sc-25778; 1:1000) was used for a loading control.

\section{Quantification and Statistics}

TIRF microscopy images were analyzed in Imaris (Oxford Instruments) software. The Spot tool was used to identify NINJ1 puncta with parameters (estimated puncta $X Y$ diameter, region type, quality filter) set 
using images from the LPS-primed group and applied to all samples across all experiments. Cells were contoured using the surfaces tool to measure cell area. The density of NINJ1 puncta was calculated on a per-cell basis by dividing the total number of puncta within the contoured plasma membrane area. Statistical testing was calculated using Prism 9.0 (GraphPad Software Inc, La Jolla, USA). Experiments with more than two groups were tested using ANOVA with Tukey's multiple comparison test. Unless otherwise indicated, presented data are representative of at least three independent experiments and are provided as mean \pm SEM.

\section{Animal studies}

All animal studies were approved by the Hospital for Sick Children Animal Care Committee.

Author contributions: Co-first authors JPB and AV conducted experiments, acquired and analyzed data, and assisted in writing the manuscript. They contributed equally and are listed alphabetically. BK conducted experiments and acquired data. NMG designed research studies, analyzed data, and assisted in writing the manuscript. BES supervised the project, designed research studies, analyzed data, and wrote the manuscript.

Acknowledgements: This work was supported by a Mentored Research Award from the International Anesthesia Research Society and an Early Investigator Award from the Department of Anesthesiology \& Pain Medicine, University of Toronto to BES. We thank Dr Nobuhiko Kayagaki and Dr Vishva Dixit for providing the rabbit monoclonal anti-mouse NINJ1 antibody, Dr Jonathan Kagan for providing the immortalized bone marrow derived macrophages expressing Cas9, and Dr John Brumell for providing pneumolysin. 


\section{References:}

1. Weinberg JM, Davis JA, Abarzua M, Rajan T. Cytoprotective effects of glycine and glutathione against hypoxic injury to renal tubules. J Clin Invest 1987;80(5):1446-1454.

2. Weinberg JM, Bienholz A, Venkatachalam MA. The role of glycine in regulated cell death. Cell Mol Life Sci 2016;73(11-12):2285-2308.

3. Loomis WP, den Hartigh AB, Cookson BT, Fink SL. Diverse small molecules prevent macrophage lysis during pyroptosis. Cell Death Dis 2019;10(4):326.

4. Volchuk A, Ye A, Chi L, Steinberg BE, Goldenberg NM. Indirect regulation of HMGB1 release by gasdermin D. Nat Commun 2020;11(1):4561.

5. Estacion M, Weinberg JS, Sinkins WG, Schilling WP. Blockade of maitotoxin-induced endothelial cell lysis by glycine and L-alanine. Am J Physiol Cell Physiol 2003;284(4):C1006-1020.

6. Weinberg JM, Buchanan DN, Davis JA, Abarzua M. Metabolic aspects of protection by glycine against hypoxic injury to isolated proximal tubules. J Am Soc Nephrol 1991;1(7):949-958.

7. Weinberg JM, Davis JA, Roeser NF, Venkatachalam MA. Role of intracellular pH during cytoprotection of proximal tubule cells by glycine or acidosis. J Am Soc Nephrol 1994;5(6):1314-1323.

8. Chen J et al. Loss of cytoskeletal support is not sufficient for anoxic plasma membrane disruption in renal cells. Am J Physiol 1997;272(4 Pt 1):C1319-1328.

9. Xia S et al. Gasdermin D pore structure reveals preferential release of mature interleukin-1. Nature 2021;593(7860):607-611. 
10. Del Re DP, Amgalan D, Linkermann A, Liu Q, Kitsis RN. Fundamental Mechanisms of Regulated Cell Death and Implications for Heart Disease. Physiol Rev 2019;99(4):1765-1817.

11. Kayagaki $\mathrm{N}$ et al. NINJ1 mediates plasma membrane rupture during lytic cell death. Nature 2021;591(7848):131-136.

12. Silva MT. Secondary necrosis: the natural outcome of the complete apoptotic program. FEBS Lett 2010;584(22):4491-4499.

13. Araki T, Zimonjic DB, Popescu NC, Milbrandt J. Mechanism of homophilic binding mediated by ninjurin, a novel widely expressed adhesion molecule. J Biol Chem 1997;272(34):21373-21380.

14. Araki T, Milbrandt J. Ninjurin, a novel adhesion molecule, is induced by nerve injury and promotes axonal growth. Neuron 1996;17(2):353-361.

15. Jeon S et al. Anti-Inflammatory Actions of Soluble Ninjurin-1 Ameliorate Atherosclerosis. Circulation 2020;142(18):1736-1751.

16. Kim S-W et al. Ninjurin 1 dodecamer peptide containing the $\mathrm{N}$-terminal adhesion motif (N-NAM) exerts proangiogenic effects in HUVECs and in the postischemic brain. Sci Rep 2020;10(1):16656.

17. Yang $\mathrm{HJ}$ et al. Ninjurin 1 has two opposing functions in tumorigenesis in a p53-dependent manner. Proc Natl Acad Sci U S A 2017;114(43):11500-11505.

18. Bae S-J et al. Ninjurin1 Assembles Into a Homomeric Protein Complex Maintained by N-linked Glycosylation. J Cell Biochem 2017;118(8):2219-2230.

19. Bertheloot D, Latz E, Franklin BS. Necroptosis, pyroptosis and apoptosis: an intricate game of cell death. Cell Mol Immunol 2021;18(5):1106-1121. 
bioRxiv preprint doi: https://doi.org/10.1101/2021.12.12.471765; this version posted December 12,2021 . The copyright holder for this preprint

(which was not certified by peer review) is the author/funder, who has granted bioRxiv a license to display the preprint in perpetuity. It is made available under aCC-BY-NC-ND 4.0 International license.

20. Flores-Romero H, Ros U, Garcia-Saez AJ. Pore formation in regulated cell death. EMBO J

2020;39(23):e105753.

21. Chen $\mathrm{X}$ et al. Translocation of mixed lineage kinase domain-like protein to plasma membrane leads to necrotic cell death. Cell Res 2014;24(1):105-121.

22. Evavold CL et al. The Pore-Forming Protein Gasdermin D Regulates Interleukin-1 Secretion from Living Macrophages.. Immunity 2018;48(1):35-44.e6. 


\section{Figures}

Figure 1. NINJ1 knockout functionally and morphologically phenocopies glycine cytoprotection. (A) Immunoblot analysis demonstrating NINJ1 knockout in iBMDM macrophages. GAPDH is presented as a loading control. (B-C) Wildtype and NINJ1 knockout iBMDM were induced to undergo pyroptosis (LPS + nigericin), secondary necrosis (venetoclax) or necrosis (pneumolysin) with or without $5 \mathrm{mM}$ glycine treatment. Cytotoxicity was evaluated by measuring the levels of LDH in the supernatant. Cytotoxicity decreased in glycine-treated wildtype cells comparably to NINJ1 knockout across each of (B) pyroptosis, (C) secondary necrosis, and (D) necrosis. Glycine treatment of NINJ1 KO cells provided no additional protection to knockout cells treated without glycine. Data are expressed as supernatant LDH as a \% of total LDH from lysates and supernatants, from $n=3$ independent experiments for each cell death type. Individual data points are shown along with their mean. ${ }^{*} P<0.05$ by ANOVA with Tukey's multiple comparison correction. (E) LPS-primed wildtype iBMDM induced to undergo pyroptosis in the presence of glycine demonstrate similar plasma membrane ballooning to NINJ1 knockout iBMDM induced to undergo pyroptosis. Membrane ballooning is shown in live cells labelled with the plasma membrane dye FM1-43. The corresponding brightfield image is shown in the inset. Scale bar $15 \mu \mathrm{m}$.

Figure 2. Glycine targets NINJ1 oligomerization to prevent membrane rupture. (A) Primary BMDM were induced to undergo pyroptosis (nigericin), apoptosis (venetoclax) or necrosis (pneumolysin) with or without glycine treatment. Native-PAGE analysis of endogenous NINJ1 demonstrates a shift to high molecular weight aggregate upon cell death stimulation, which is abrogated by glycine treatment. (B) Pyroptosis was induced in primary LPS-primed BMDM with nigericin with or without glycine. Immunofluorescence microscopy of native NINJ1 (green) reveals a redistribution of NINJ1 from diffuse plasma membrane staining to discrete puncta. NINJ1 does not redistribute in glycine-treated cells. Nuclei are labeled with DAPI (blue). Scale bar $15 \mu \mathrm{m}$. Inset shows magnified area demarcated by the white box. (C) Total internal reflection microscopy of NINJ1 in LPS-primed primary BMDM reveals that endogenous NINJ1 resides in discrete puncta within the plasma membrane. Cell membrane outline (dotted white line) was determined using fluorescently labeled cholera toxin subunit B (not shown) as a plasma membrane marker. Scale bar $20 \mu \mathrm{m}$. Inset shows magnified area demarcated by the white box. (D) Quantification of the density of NINJ1 puncta in LPS-primed primary macrophages at baseline or stimulated to undergo pyroptosis (nigericin $20 \mu \mathrm{M}$ for $30 \mathrm{~min}$ ) without or with glycine (5 mM). NINJ1 puncta become less dense upon pyroptosis induction, consistent with NINJ1 plasma membrane clustering. Glycine limits this redistribution. Violin plot of NINJ1 puncta density from 3 pooled independent experiments. Data points superimposed on the violin plots are the mean NINJ1 puncta densities for the 3 independent experiments (10-23 cells measured per replicate with >675 NINJ1 puncta identified per replicate). Bars represent mean \pm SEM of the NINJ1 densities for the $n=3$ independent experiments. $* P<0.05$ by ANOVA with Tukey's multiple comparison correction. 
bioRxiv preprint doi: https://doi.org/10.1101/2021.12.12.471765; this version posted December 12, 2021. The copyright holder for this preprint (which was not certified by peer review) is the author/funder, who has granted bioRxiv a license to display the preprint in perpetuity. It is made available under aCC-BY-NC-ND 4.0 International license.

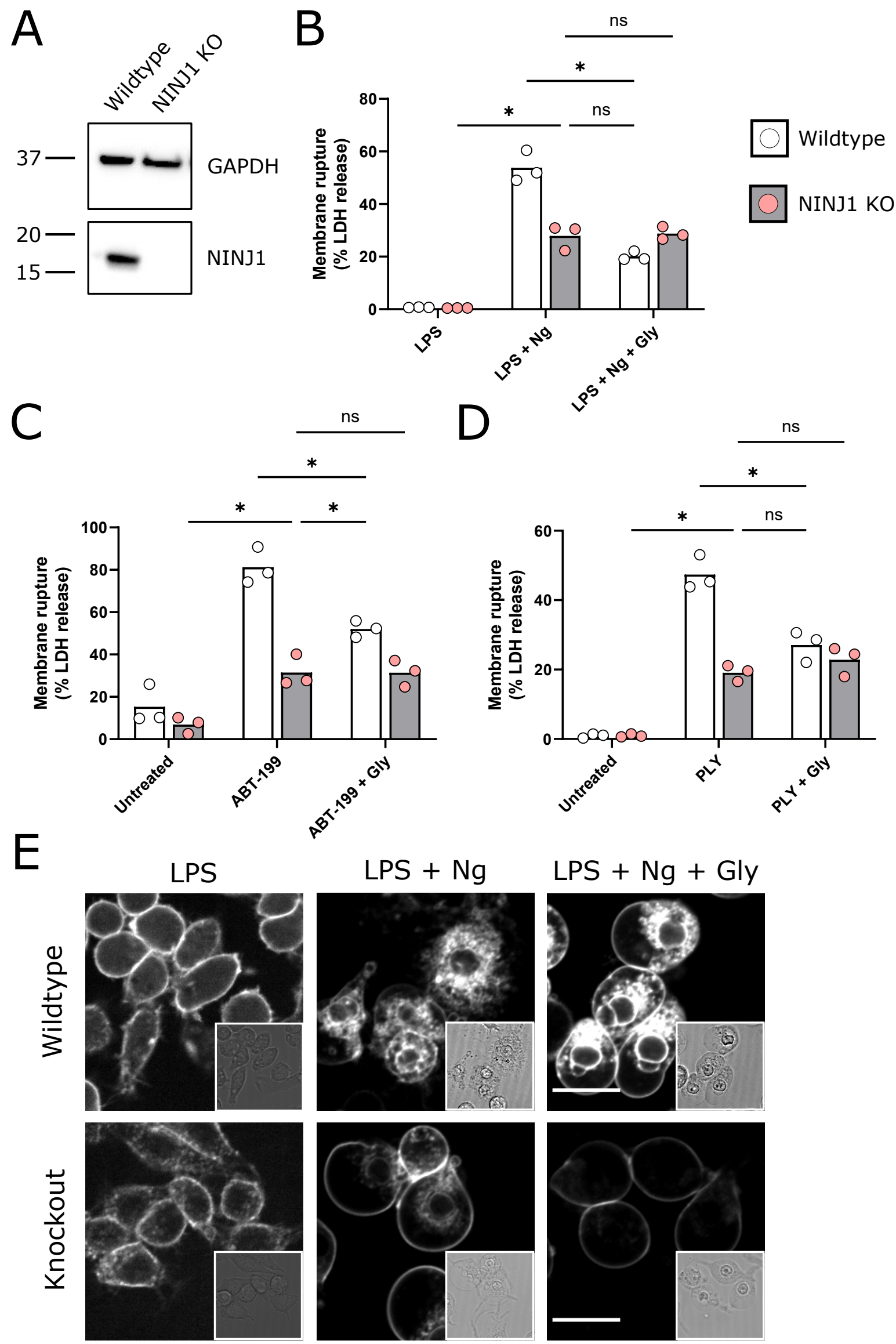



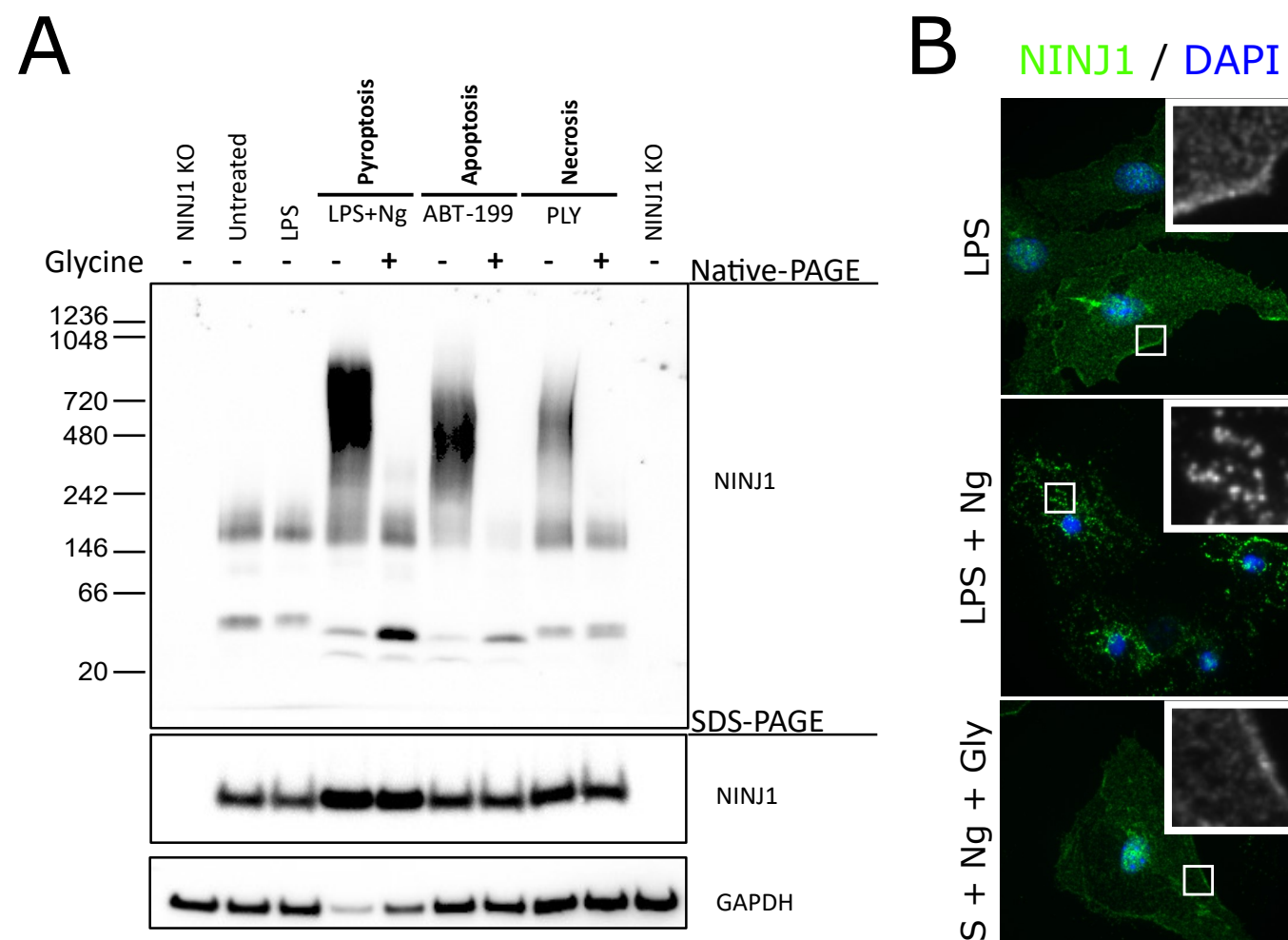

$\mathcal{C}$
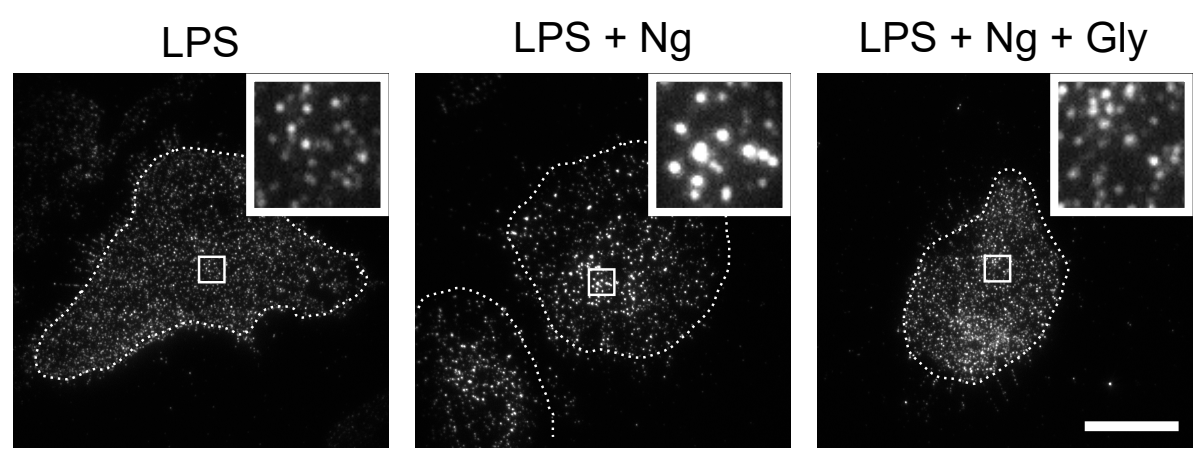

D

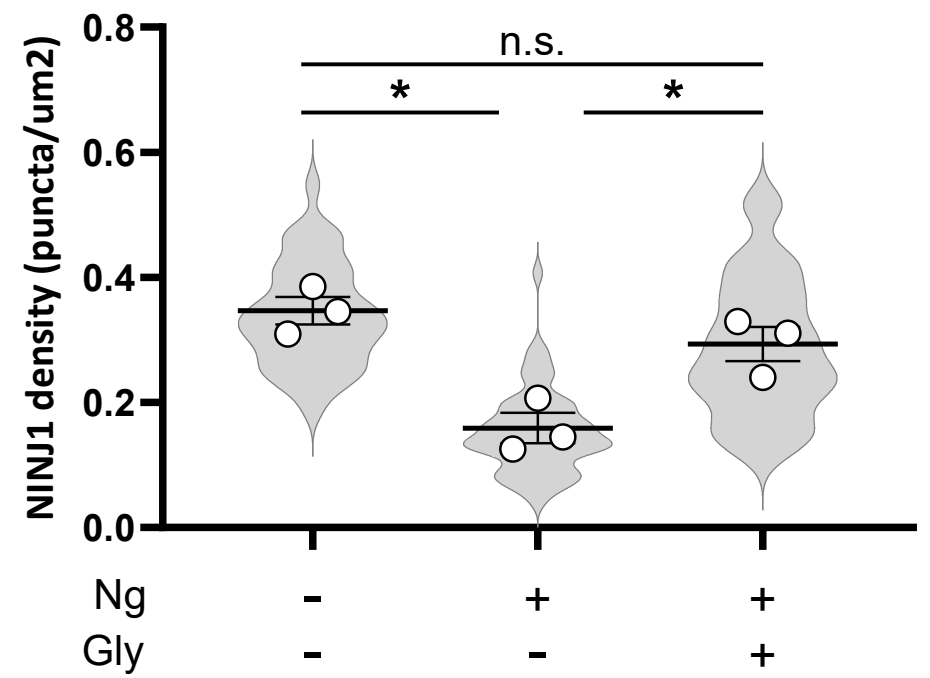

